\title{
Microtensile Bond Strength of Composite Cement to Novel CAD/ CAM Materials as a Function of Surface Treatment and Aging
}

DP Lise • A Van Ende • J De Munck • LCC Vieira • LN Baratieri • B Van Meerbeek

\section{Clinical Relevance}

Novel composite and polymer-infiltrated ceramic CAD/CAM materials benefit from etching of the intaglio surface with hydrofluoric acid or sandblasting, both followed by silanization.

\section{SUMMARY}

Objectives: To evaluate the effect of different surface treatments on the bond strength to a

Diogo Pedrollo Lise, DDS, MSD, PhD, Department of Operative Dentistry, Federal University of Santa Catarina (UFSC), Santa Catarina, Brazil

Annelies Van Ende, DDS, MSD, PhD, KU Leuven - BIOMAT Department of Oral Health Sciences, KU Leuven and Dentistry, University Hospitals Leuven, Leuven, Belgium

Jan De Munck, DDS, MSD, PhD, postdoctoral research fellow, KU Leuven - BIOMAT, Department of Oral Health Sciences, KU Leuven and Dentistry, University Hospitals Leuven, Leuven, Belgium

Luiz Clovis Cardoso Vieira, DDS, MSD, PhD, professor emeritus, Department of Operative Dentistry, Federal University of Santa Catarina (UFSC), Santa Catarina, Brazil

Luiz Narciso Baratieri, DDS, MSD, PhD, professor emeritus, Department of Operative Dentistry, Federal University of Santa Catarina (UFSC), Santa Catarina, Brazil

*Bart Van Meerbeek, DDS, MSD, PhD, full professor, KU Leuven - BIOMAT, Department of Oral Health Sciences, KU Leuven and Dentistry, University Hospitals Leuven, Leuven, Belgium

*Corresponding author: Kapucijnenvoer 7, Leuven 3000, Belgium; e-mail: bart.vanmeerbeek@kuleuven.be

DOI: $10.2341 / 15-263-\mathrm{L}$ composite and a polymer-infiltrated ceramic CAD/CAM block after six-month artificial aging.

Methods and Materials: Two types of CAD/CAM blocks (Cerasmart, GC; Enamic, Vita Zahnfabrik) were cut in slabs of 4-mm thickness, divided into six groups, and subjected to the following surface treatments: group 1: no treatment; group 2: sandblasting (SB); group 3: SB + silane (Si); group 4: SB + Si + flowable composite (see below); group 5: $5 \%$ hydrofluoric acid etching (HF) + Si; and group 6: $37 \%$ phosphoric acid etching $\left(\mathrm{H}_{3} \mathrm{PO}_{4}\right)+\mathrm{Si}$. Sections of the same group were luted together $(n=3: 3$ sandwich specimens/group) using a dual-cure self-adhesive cement for all groups, except for the sections of group 4 that were luted using a light-curing flowable composite. After three weeks of storage in $0.5 \%$ chloramine at $37^{\circ} \mathrm{C}$, the sandwich specimens were sectioned in rectangular microspecimens and trimmed at the interface to a dumbbell shape (1.1-mm diameter). One half of the specimens was subjected to a microtensile bond strength $(\mu$ TBS) test, and the other half was tested after six months of water storage (aging). Data were 
statistically analyzed with a linear mixed-effects model for the factors surface treatment, material type, and aging, together with their first-degree interactions $(\alpha=0.05)$.

Results: The lowest bond strengths were obtained in the absence of any surface treatment (group 1), while the highest $\mu$ TBSs were obtained when the surface was roughened by either SB or HF, this in combination with chemical adhesion through Si. Loss in bond strength was observed after six-month aging when either surface roughening or silanization, or both, were omitted.

Conclusions: Both the composite and polymerinfiltrated ceramic CAD/CAM blocks appeared equally bonding-receptive regardless of the surface treatment used. Creating a microretentive surface by either SB or HF, followed by chemical adhesion using $\mathrm{Si}$, is mandatory to maintain the bond strength after six months.

\section{INTRODUCTION}

Chairside CAD/CAM restorations have become increasingly popular in the latest decade thanks to recent improvements in $\mathrm{CAD} / \mathrm{CAM}$ technology, which increased the ease of use and cost effectiveness of the restorations. Recently, new types of blocks containing either composite or both a polymeric and ceramic phase have been developed. ${ }^{1}$ Cerasmart (GC, Tokyo, Japan) is a 'composite' block that consists of evenly dispersed ceramic nanoparticles $(71 \mathrm{vol} \%)$ in a polymeric matrix, ${ }^{2}$ providing a high flexural strength ${ }^{3}$ and modulus of resilience. ${ }^{1}$ Enamic (Vita Zahnfabrik, Bad Säckingen, Germany) is a so-called 'polymer-infiltrated ceramic' and presents a three-dimensionally interconnected pre-sintered ceramic network of $86 \mathrm{vol} \%$ that is infiltrated with a monomer mixture; thus, intertwined networks of polymers and ceramics are created. ${ }^{4-6}$ The polymeric network is thought to render the material less brittle than classic ceramics, as inferred from its higher Weibull modulus. ${ }^{7,8}$

To be clinically successful, adequate adhesion of the restoration is very important. It has been shown ${ }^{9-11}$ that surface treatment prior to cementation can enhance the bond strength to indirect restorations. Micromechanical retention can be provided through sandblasting or acid etching, while a silane coupling agent provides chemical bonding. ${ }^{11}$ Different strategies are preferred depending on the material's characteristics. ${ }^{12}$
However, information about bonding protocols to new composite and polymer-infiltrated ceramic materials is scarce. ${ }^{13-15}$ In a previous study, ${ }^{14}$ it was suggested that bonding strategies were materialdependent. Therefore, the objective of this study was to evaluate the effect of different surface treatments on the bond strength of a self-adhesive composite cement to new CAD/CAM blocks. The null hypotheses were that 1 ) material type, 2) surface treatment, and 3) aging did not have an influence on the microtensile bond strength ( $\mu \mathrm{TBS}$ ) to either a composite or polymer-infiltrated ceramic $\mathrm{CAD} / \mathrm{CAM}$ block.

\section{METHODS AND MATERIALS}

The experimental procedure is schematically illustrated in Figure 1, and all of the materials that were used are listed in Table 1. Two types of CAD/CAM blocks (Cerasmart [CER], GC; and; Enamic [ENA], Vita Zahnfabrik) of $12 \times 14 \times 18 \mathrm{~mm}$ were sectioned using a diamond blade in slabs of 4-mm thickness and wet-polished with 600 -grit silicon carbide paper for 30 seconds. Thirty-six slabs of each CAD/CAM material were selected and ultrasonically cleaned for five minutes in distilled water to remove surface contaminants. For each block type, the specimens were randomly divided into six groups of six 4-mm slabs in accordance with the surface treatment, as follows:

- Group 1: No surface treatment (NT).

- Group 2: Sandblasting (SB): the surface was sandblasted with 27- $\mu \mathrm{m}$ aluminum-oxide $\left(\mathrm{Al}_{2} \mathrm{O}_{3}\right)$ particles perpendicular to the surface from a distance of $10 \mathrm{~mm}$ over the course of 20 seconds with a pressure of $0.28 \mathrm{MPa}$. Remaining particles were removed using a gentle air-blow for five seconds.

- Group 3: Sandblasting + silane (SB/Si): the surface was sandblasted following the same protocol as in group 2, and then a thin layer of a silane coupling agent (Ceramic Primer II, GC) was applied using a disposable microtip applicator. After 60 seconds, the surface was dried with an air syringe.

- Group 4: Sandblasting + silane + flowable composite ( $\mathrm{SB} / \mathrm{Si} / \mathrm{FLO})$ : the surface treatment and silanization were carried out following the same protocol as in group 3; however, a flowable composite was used as luting agent.

- Group 5: Hydrofluoric acid etching + silane (HF/ $\mathrm{Si}$ ): the surface was etched with $5 \%$ hydrofluoric acid (HF; IPS Ceramic Etching Gel 5\%, Ivoclar Vivadent, Schaan, Liechtenstein) for 60 seconds 


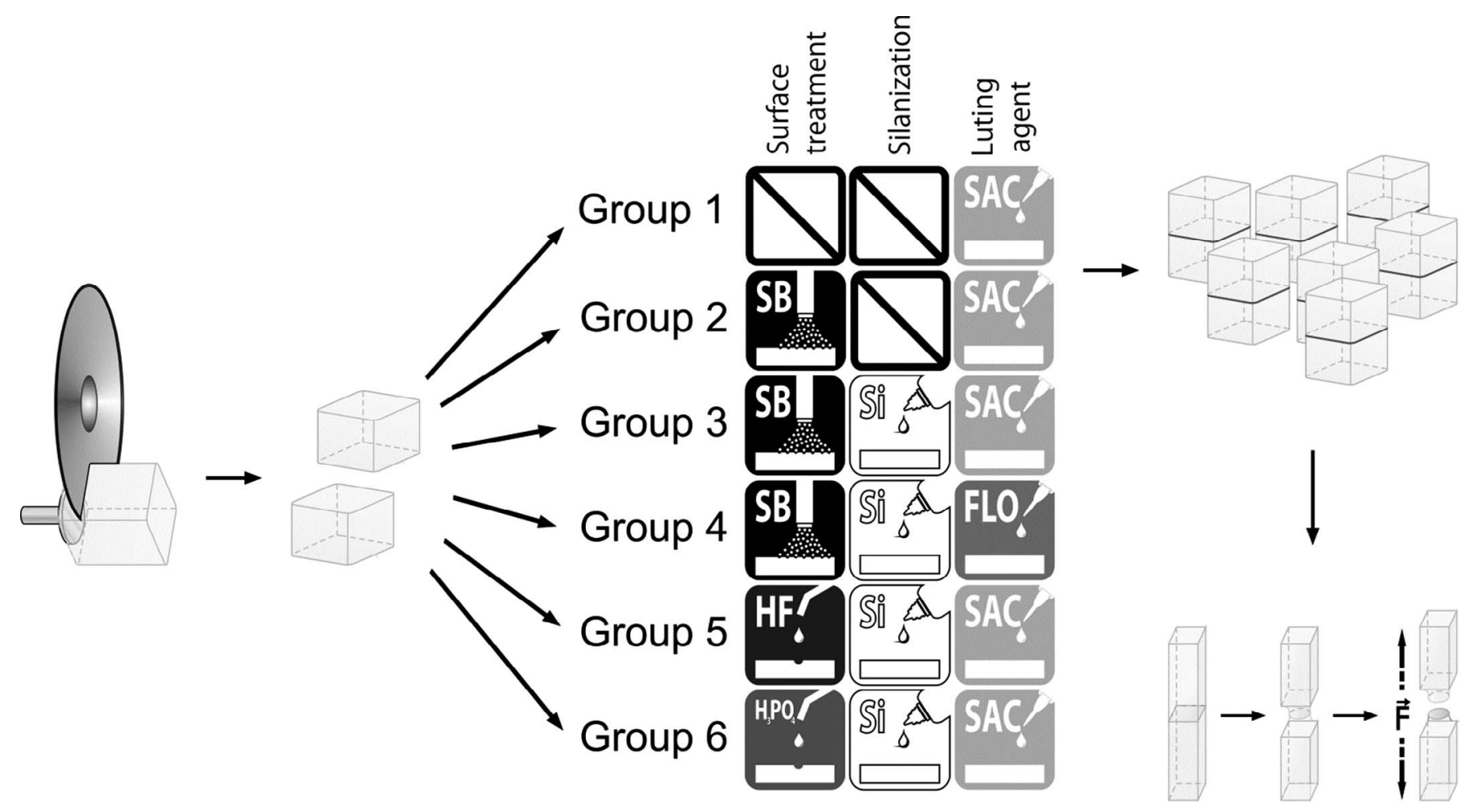

Figure 1. Scheme explaining the study set. Standardized 4-mm slabs were cut from two types of CAD/CAM block materials and filled according to the respective experimental conditions, resulting in 2 (material) $\times 6$ groups (surface treatment and silanization). Microspecimens were prepared and stressed until failure after three weeks and six months. SB: sandblasting with $27-\mu \mathrm{m} \mathrm{Al}_{2} \mathrm{O}_{3} ;$ Si: silane; $\mathrm{HF}: 5 \%$ hydrofluoric acid; $\mathrm{H}_{3} P \mathrm{O}_{4}$ : $37 \%$ phosphoric acid; SAC: self-adhesive composite cement; FLO: flowable composite.

and thoroughly rinsed by water spray for 60 seconds. Then the surface was cleaned ultrasonically in distilled water for five minutes and airdried for 20 seconds. Silane was applied following the same protocol as in group 3.

- Group 6: Phosphoric acid etching + silane $\left(\mathrm{H}_{3} \mathrm{PO}_{4} /\right.$ $\mathrm{Si})$ : surface etching with $37 \%$ phosphoric acid (Total Etch, Ivoclar Vivadent) for 60 seconds, followed by water rinsing for 60 seconds and airdrying for 20 seconds. Silane was applied following the same protocol as in group 3.

Three pairs of $4-\mathrm{mm} \mathrm{CAD} / \mathrm{CAM}$ slabs $(12 \times 14 \times 4$ $\mathrm{mm}$ ) from the same CAD/CAM block material and the same group were luted together (3 sandwich specimens per group; $n=3$ ) using a self-adhesive composite cement (G-CEM LinkAce, GC), whereas a light-cured flowable composite (G-ænial Universal Flo, GC) was used to lute the slabs of group $4(\mathrm{SB} / \mathrm{Si} /$ FLO). The cementation was performed under a constant weight of $1 \mathrm{~kg}$ over the course of 10 minutes. The excess luting agent was removed with a microtip applicator, and the cementation line was covered with a glycerin gel (Liquid Strip, Ivoclar Vivadent) in order to prevent the formation of an oxygen-inhibited layer. After the initial five minutes, the sandwich specimens were light-cured from each side for 40 seconds using a LED light-curing unit (Prima Light, GC) with an output of $\sim 1600 \mathrm{~mW} / \mathrm{cm}^{2}$, as measured by the MARC Patient Simulator (BlueLight Analytics, Halifax, NS, Canada). Specimens were stored in $0.5 \%$ chloramine solution for three weeks at $37^{\circ} \mathrm{C}$. Next, each sandwich specimen was sectioned perpendicularly to the luting interface using an automated water-cooled diamond saw (Accutom-50, Struers, Ballerup, Denmark) to obtain up to six microspecimens $(1.7 \times 1.7 \times 8 \mathrm{~mm})$. Each microspecimen was trimmed at the interface to a dumbbell shape $(1.1 \pm 0.1 \mathrm{~mm}$ diameter $)$ with a cylindrical extrafine-grit $(15 \mu \mathrm{m})$ diamond bur fixed in a water-cooled high-speed handpiece mounted in a computer-controlled lathe (MicroSpecimen Former, University of Iowa, Iowa City, IA, USA). Next, the cross-sectional diameters of the dumbbell-shaped specimens were measured with an X-Y multipurpose modular measuring microscope equipped with a digital readout (Leitz VRZ-U, Wetzlar, Germany) to an accuracy of $0.001 \mathrm{~mm}$. One half of the microspecimens were stored in $0.5 \%$ chloramine at $37^{\circ} \mathrm{C}$ for three weeks and the other half during six months before testing. Upon testing, they were attached to a 


\begin{tabular}{|c|c|c|c|}
\hline \multicolumn{2}{|c|}{ Materials } & \multirow{2}{*}{$\begin{array}{l}\text { Composition } \\
\text {-Silica }(20 \mathrm{~nm}) \text { and barium glass }(300 \mathrm{~nm}) \\
\text { nanoparticles }(71 \mathrm{wt} \%) \\
\text { •Polymers }(29 \%) \text { of Bis-MEPP, UDMA, and } \\
\text { DMA }\end{array}$} & \multirow{2}{*}{$\frac{\text { Batch No }}{1403101}$} \\
\hline Composite CAD/CAM block & Cerasmart, GC, Tokyo, Japan & & \\
\hline Polymer-infiltrated CAD/CAM block & $\begin{array}{l}\text { Enamic, Vita Zahnfabrik, Bad } \\
\text { Säckingen, Germany }\end{array}$ & $\begin{array}{l}\text {-Feldspar ceramic reinforced by oxides ( } 86 \\
\text { wt.\%) } \\
\text { •Polymers (14\%) of UDMA and TEGDMA } \\
\end{array}$ & 53570 \\
\hline \multirow[t]{2}{*}{ Luting agents } & G-CEM LinkAce, GC & $\begin{array}{l}\text {-Paste A: UDMA } 10 \%-20 \%, Y \text { - } \\
\text { methacryloxypropyltrimethoxysilane }>2.5 \% \\
\text { •Paste B: UDMA } 25 \%-50 \% \text {, } \\
\text { methacryloxypropyltrimethoxysilane }>2.5-10 \% \text {, } \\
\text { A, } \alpha \text {-dimethylbenzylhydroperoxide }\end{array}$ & 1401151 \\
\hline & G-ænial Universal Flo, GC & $\begin{array}{l}\text {-Matrix: UDMA, Bis-MEPP, TEGDMA } \\
\text {-Fillers (69 wt.\%): silicon dioxide (16 nm) and } \\
\text { strontium glass }(200 \mathrm{~nm}) \\
\text { •Others: pigments, photoinitiator }\end{array}$ & 1312072 \\
\hline Hydrofluoric acid (HF) & $\begin{array}{l}\text { IPS Ceramic Etching Gel 5\%, } \\
\text { Ivoclar Vivadent, Schaan, } \\
\text { Liechtenstein }\end{array}$ & Aqueous solution of hydrofluoric acid $(<5 \%)$ & S51072 \\
\hline Phosphoric acid $\left(\mathrm{H}_{3} \mathrm{PO}_{4}\right)$ & Total Etch $37 \%$, Ivoclar Vivadent & Phosphoric acid (37\%), water, & S29080 \\
\hline Sandblasting particles & $\begin{array}{l}\text { Aluminum-oxide } 27-\mu \mathrm{m} \text {, Danville } \\
\text { Materials, San Ramon, CA, USA }\end{array}$ & Aluminum-oxide $27-\mu \mathrm{m}$ particles & 28482 \\
\hline
\end{tabular}

notched BIOMAT jig $^{16}$ with cyanoacrylate glue (Model Repair II Blue, Dentsply-Sankin, Tochigiken, Japan) and stressed until failure in a universal testing device (Instron 5848 Micro Tester, High Wycombe, Bucks, UK) at a crosshead speed of 1 $\mathrm{mm} / \mathrm{min}$, using a load cell of $500 \mathrm{~N}$. When a specimen broke during the trimming procedure, it was recorded as a pretesting failure (ptf). Microtensile bond strength data ( $\mu$ TBS per microspecimen in $\mathrm{MPa}$ ) were statistically analyzed using a linear mixed-effects model. Fixed effects included in the model were the factors 'surface treatment' (groups 1 to 6), 'material type' (CER vs ENA), and 'aging' or storage period (three weeks vs six months), along with their first-degree interactions. The sandwich blocks were added to the model as a random factor to account for the multiple testings per block. In addition, to evaluate the six-month results more profoundly, specific contrasts, along with a $p$-value corrected for the amount of tests, were calculated in order to compare the six-month results to their respective negative (group 1: NT) and positive (group 3: $\mathrm{SB} / \mathrm{Si}$ ) control. Group $3(\mathrm{SB} / \mathrm{Si})$ was selected as the positive control as a result of the considerable polymer phase of both materials. All tests were performed at a significance level of $\alpha=0.05$ using a software package. ${ }^{17}$

The mode of failure was assessed using scanning electron microscopy (SEM; JSM-6610LV SEM, Jeol, Tokyo, Japan) at a magnification of $70 \times$, and failures were classified as either 'interfacial' (between the luting agent and the CAD/CAM block material and/ or within the luting agent) or 'mixed' (involvement of both interfacial fracture and cohesive fracture within the CAD/CAM block substrate). Additional CAD/CAM block slabs were prepared following the previous surface treatment protocols (NT, SB, HF, and $\mathrm{H}_{3} \mathrm{PO}_{4}$ ) in order to morphologically analyze the surface topography using SEM. Specimens were mounted on aluminum stubs with adhesive carbon tape (PELCO Carbon Conductive Tape, Ted Pella Inc, Redding, CA, USA) and sputter-coated with gold-palladium by means of a sputter-coater (JFC1300 Autofine Coater, Jeol) under a chamber pressure of $30 \mathrm{~mA} / \mathrm{Pa}$ for 120 seconds. Specimens 


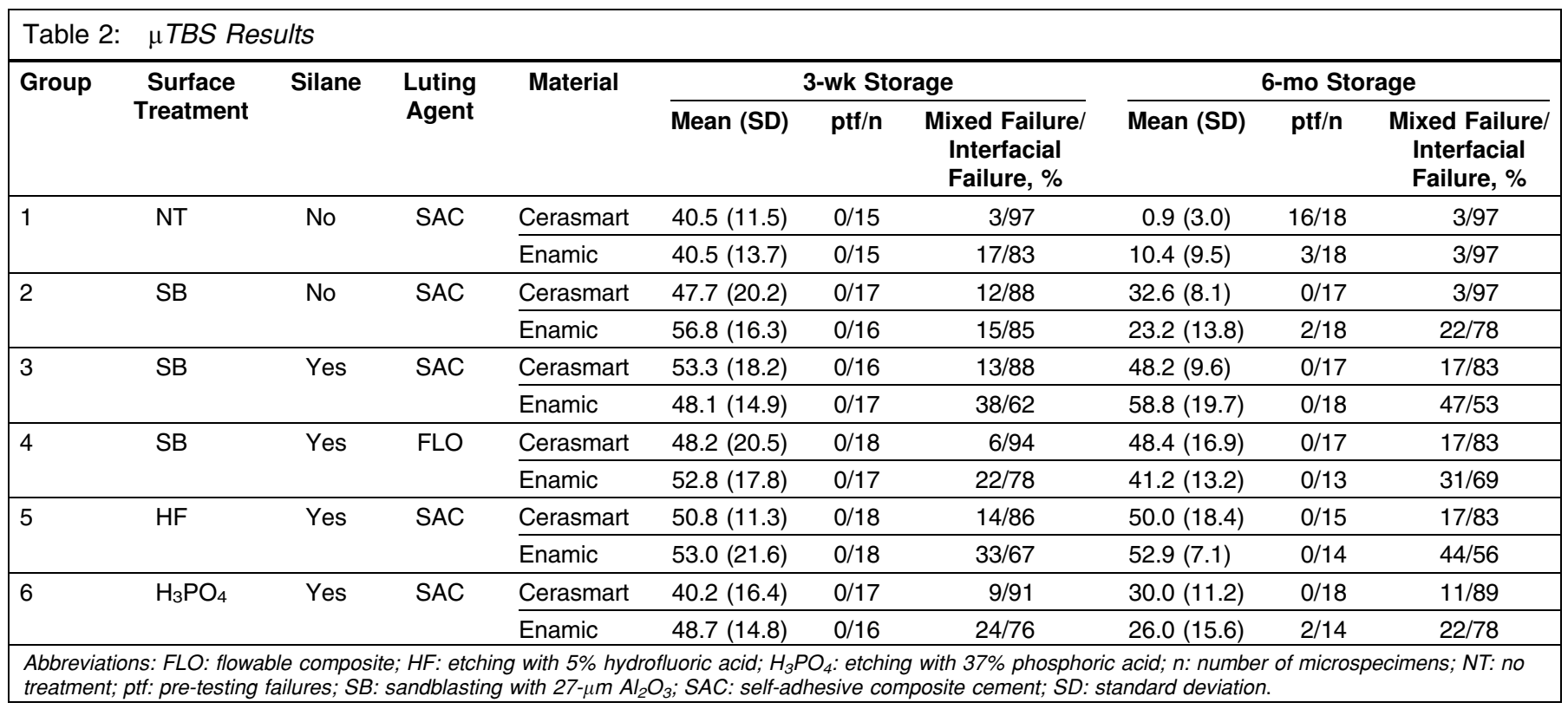

were observed under SEM at an accelerating voltage of $15 \mathrm{kV}$ and a working distance of $11.0 \mathrm{~mm}$.

\section{RESULTS}

The lowest bond strengths were obtained in the absence of any surface treatment (Table 2). No effect was found for the 'material type' $(p=0.97)$, while significant effects of the factors 'surface treatment' $(p<0.0001)$ and 'aging' $(p<0.0001)$ were found. A highly significant interaction between surface treatment and aging $(p<0.0001)$ was found as well. After three-week storage, there were no significant differences in $\mu$ TBS values between the experimental groups. After six months, any surface treatment (groups 2-6) resulted in significantly higher bond strengths than were noted in the negative control (group 1: NT), which didn't receive any treatment (Figure 2). However, when silanization was omitted (group 2: SB), the results were significantly lower than those of the positive control (group 3: $\mathrm{SB} / \mathrm{Si}$ ) when both sandblasting and silanization were applied. Substituting the sandblasting for etching with $\mathrm{HF}$ (group 5: $\mathrm{HF} / \mathrm{Si}$ ) or substituting the self-adhesive composite cement for a flowable composite (group 4: $\mathrm{SB} / \mathrm{Si} / \mathrm{FLO}$ ) rendered results that were not significantly different from that of the positive control (group 3: SB/Si). In contrast, substituting the sandblasting for etching with $\mathrm{H}_{3} \mathrm{PO}_{4}$ (group 6: $\mathrm{H}_{3} \mathrm{PO}_{4} / \mathrm{Si}$ ) was not sufficient and resulted in a significant decrease in bond strength in comparison with that of the positive control (group 3: $\mathrm{SB} / \mathrm{Si}$ ).
SEM analysis of the surface treatments demonstrated that the untreated surface of CER had a smoother appearance than that of ENA. While roughening effects could be seen for both types of CAD/CAM blocks after $\mathrm{SB}$ and $\mathrm{HF}$ (Figure 3), surface treatment with $\mathrm{H}_{3} \mathrm{PO}_{4}$ did not result in a visible morphological difference. $\mathrm{SB}$ resulted in an irregular, rugged surface in both $\mathrm{CAD} / \mathrm{CAM}$ block materials, while HF created porelike holes in the surface of CER, having dissolved the silica and barium-glass nanoparticles, but resulted in a more rugged appearance when dissolving the feldspathic ceramic network of ENA (Figure 3). Failure analysis demonstrated a higher prevalence of mixed fractures with ENA than with CER (Table 2; Figure 4).

\section{DISCUSSION}

This in vitro study was designed to investigate the effect of various surface treatments on the adhesion of a dual-cure, self-adhesive composite cement to two novel CAD/CAM block materials after two storage times. Despite inherent differences in surfaces (Figure 3), no differences in bond strength to CER vs ENA were found (Table 2); thus, the first null hypothesis was accepted. Qualitative analysis using SEM showed that the untreated surface of ENA was rougher than the surface of CER (Figure 3); this might explain why there were fewer pretesting failures in ENA/NT than in CER/NT after six months (Table 2), although this difference was not significantly different $(p=0.084)$. 


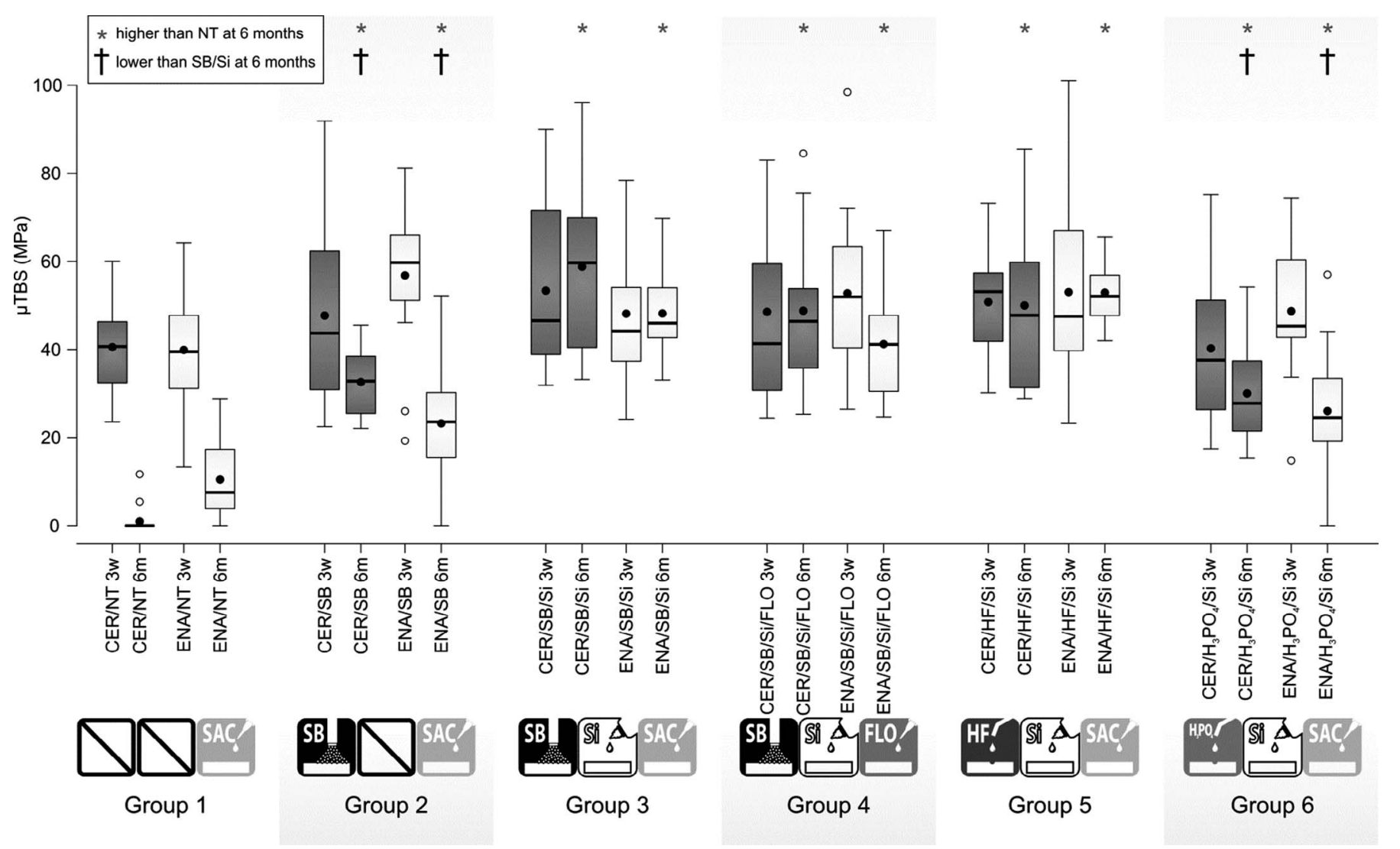

Figure 2. Boxplots of the $\mu$ TBS results. The box represents the spreading of the data between the first and third quartile. The central horizontal line and the black dot represent the median and mean, respectively. The whiskers extend to the minimum and maximum values measured, with the exception of the outliers that are represented with open dots $\rho)$. CER: Cerasmart; ENA: Enamic; NT: no treatment; SB: sandblasting with 27- $\mu$ m $\mathrm{Al}_{2} \mathrm{O}_{3}$; Si: silane; HF: $5 \%$ hydrofluoric-acid etching; $\mathrm{H}_{3} \mathrm{PO}_{4}$ : $37 \%$ phosphoric-acid etching; SAC: self-adhesive composite cement; $\mathrm{FLO}$ : flowable composite. Significant differences are based on linear mixed-effects models at a significance level of $\mathrm{p}=0.05$.
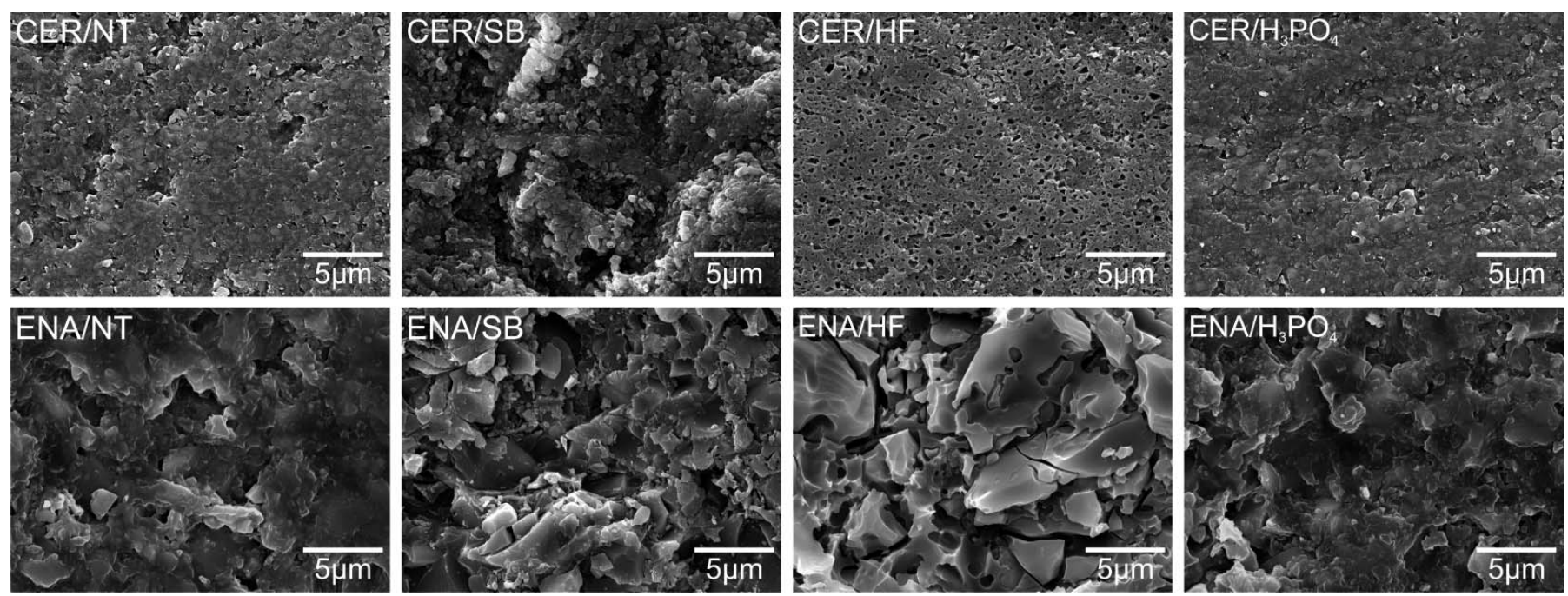

Figure 3. SEM photomicrographs of pretreated surfaces. CER: Cerasmart (GC); ENA: Enamic (Vita Zahnfabrik); NT: no treatment; SB: sandblasting with $27-\mu \mathrm{m} \mathrm{Al}{ }_{2} \mathrm{O}_{3}$; HF: 5\% hydrofluoric-acid etching; $\mathrm{H}_{3} \mathrm{PO}_{4}$ : 37\% phosphoric-acid etching. Original magnification: $5000 \times$. 
3 weeks
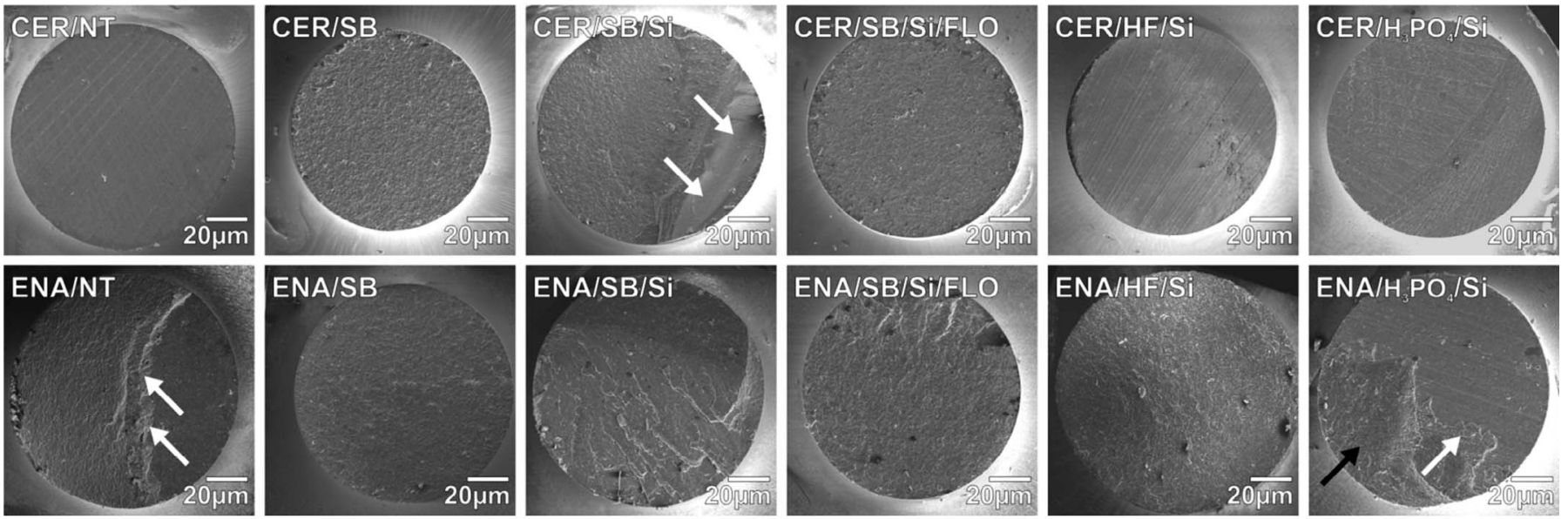

\section{6 months}
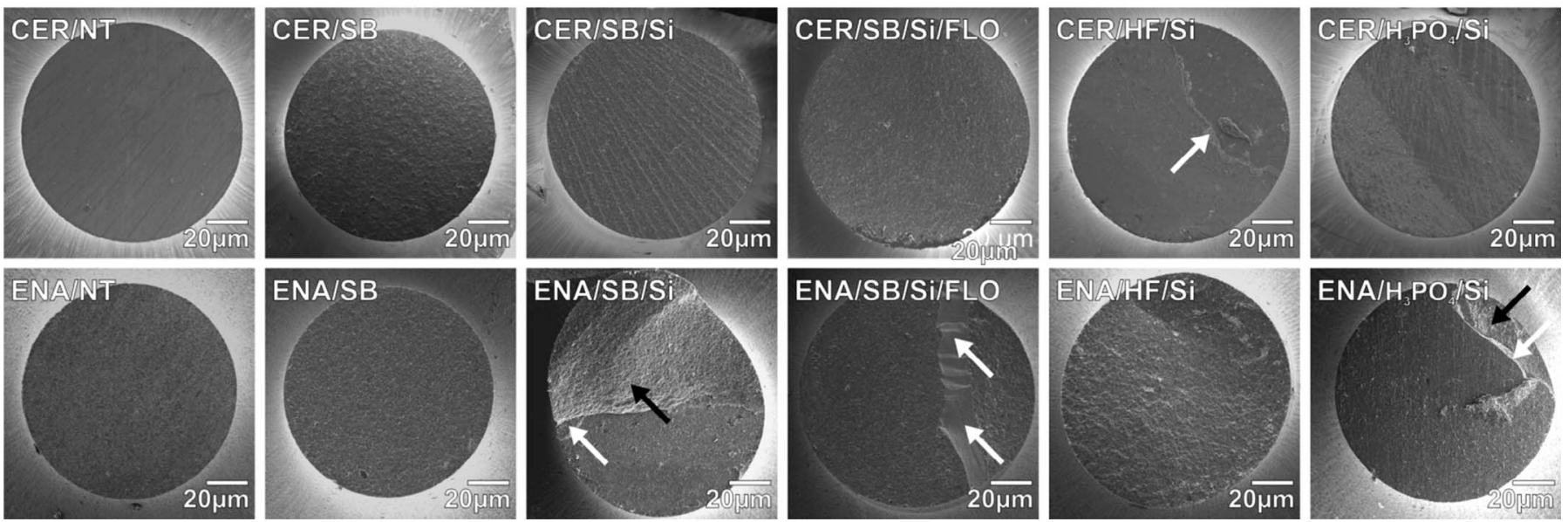

Figure 4. SEM photomicrographs of $\mu$ TBS-fractured surfaces. CER: Cerasmart (GC); ENA: Enamic (Vita Zahnfabrik); NT: no treatment; SB: sandblasting with 27- $\mu \mathrm{m} \mathrm{Al} \mathrm{O}_{3}$; HF: $5 \%$ hydrofluoric-acid etching; $\mathrm{H}_{3} \mathrm{PO}_{4}$ : 37\% phosphoric-acid etching; Si: silane; FLO: flowable composite. White arrows: interfacial failure at the composite cement. Black arrows: mixed failure with involvement of CAD/CAM block substrate. Note that more mixed failures occurred within the CAD/CAM block substrate with ENA than with CER. Original magnification: 70X.

It must be noted that the CER used in this study contains etchable barium-glass particles and has a lower hardness ${ }^{3}$ as opposed to the zirconia-containing composite $\mathrm{CAD} / \mathrm{CAM}$ block material used in previous studies. ${ }^{14,15}$ As a consequence, CER is more susceptible to mechanical roughening and acid etching. ${ }^{18}$ It is clear that differences within material classes must be interpreted with caution ${ }^{19}$ and that those findings cannot be generalized to all composite CAD/CAM block materials.

Both surface treatment and storage period (aging) had a significant influence on bond strength, with a significant interaction between both factors $(p<0.001)$; hence, the second and third null hypotheses were rejected. Overall, high levels of bond strength were found after only three weeks of water storage (Table 2). Differences between the surface treatments only manifested after a longer water storage period, which affected the bond strength, depending on the surface treatment. The lowest bond strengths were obtained in the absence of any surface treatment (group 1: NT), which became apparent after six-month storage. After this period of six months, any surface treatment resulted in a higher bond strength in comparison with the negative control NT (Figure 2). However, when compared to group $3(\mathrm{SB} / \mathrm{Si})$, which served as a positive control, SB alone (group 2) resulted in significantly lower bond strengths (Figure 2). This shows that the silanization step is crucial to maintain an optimal adhesion, which was also demonstrated for conventional ceramics ${ }^{20,21}$ and 
indirect composites. ${ }^{22,23}$ Despite the presence of polymers in both CAD/CAM block materials, their inorganic content remained relatively high so that the extra coupling provided by the silane between the inorganic and polymer constituents of the luting agent enhanced the bond strength. ${ }^{24}$ However, mechanical roughening is also necessary; in group $6\left(\mathrm{H}_{3} \mathrm{PO}_{4} / \mathrm{Si}\right), \mathrm{H}_{3} \mathrm{PO}_{4}$ was not strong enough to induce visible surface roughening, and despite silanization lower bond strengths were obtained than in the positive control group $3(\mathrm{SB} / \mathrm{Si})$. Similar results have been found for conventional ceramics ${ }^{11,25}$ composites ${ }^{26}$ and polymer-infiltrated ceramics. ${ }^{27}$ Surface roughness of the $\mathrm{H}_{3} \mathrm{PO}_{4}$-etched surface was similar to that of the untreated surface, but it is thought to have a cleaning effect. ${ }^{18,27} \mathrm{H}_{3} \mathrm{PO}_{4}$ might be preferred over $\mathrm{HF}$ as a surface treatment for intraoral repair because of the potential hazards of the latter material ${ }^{28}$; however, based on these results, it can be concluded that bonding in the long term might be compromised in the absence of sufficient micromechanical retention.

Fracture analysis revealed that the majority of specimens failed at the interface, which indicates that the stress was concentrated in this area during the tensile test. ${ }^{29}$ More mixed failures, with large parts of cohesive fractures in the substrate, were seen for ENA; this might be a result of the inherent higher brittleness of this material in comparison with the more resilient CER. Indeed, it has been shown ${ }^{1}$ that ENA has a lower flexural strength than CER. Flexural strength is closely related to tensile strength, and this might explain why failures propagated more often through the substrate in ENA.

A dual-cure, self-adhesive composite cement (GCEM LinkAce, GC) was used to lute all of the specimens, except for those of group $4(\mathrm{SB} / \mathrm{Si} / \mathrm{FLO})$, for which a light-curing flowable composite (G-ænial Universal Flo) was used as luting agent. Interestingly, the latter was found to be equally effective as the dual-cure composite cement with both CAD/CAM block materials (Figure 2). It must be kept in mind, however, that in this case the sandwich specimens were extensively light-cured from each side and that as a result of the flat interface of the specimens, a beneficial ratio between the circumference-which can be exposed directly to the light-and intaglio surface was created. To extrapolate this finding to clinical situations, however, more studies regarding the degree of conversion of these composites under composite/ceramic restorations of various thicknesses are necessary.

\section{CONCLUSIONS}

Both the composite and polymer-infiltrated ceramic blocks performed equally well regardless of surface treatment. As previously shown for other indirect $\mathrm{CAD} / \mathrm{CAM}$ materials, ${ }^{13,20,30-33}$ creating a microretentive surface by either sandblasting or hydrofluoric-acid etching, followed by silanization for chemical adhesion, is mandatory to maintain the bond strength upon water storage for six months.

\section{Acknowledgements}

This investigation was supported in part by the Brazilian Agency for Support and Evaluation of Graduate EducationCAPES (grant BEX 13930/13-3). The dental companies GC, Vita Zahnfabrik, and Ivoclar Vivadent are gratefully acknowledged for the generous donation of materials for this study.

\section{Conflict of Interest}

The authors of this manuscript certify that they have no proprietary, financial, or other personal interest of any nature or kind in any product, service, and/or company that is presented in this article.

(Accepted 9 May 2016)

\section{REFERENCES}

1. Awada A, \& Nathanson D (2015) Mechanical properties of resin-ceramic CAD/CAM restorative materials Journal of Prosthetic Dentistry 114(4) 587-593, doi:10.1016/j. prosdent.2015.04.016.

2. Lauvahutanon S, Takahashi H, Oki M, Arkornnukit M, Kanehira M, \& Finger WJ (2015) In vitro evaluation of the wear resistance of composite resin blocks for CAD/ CAM Dental Materials Journal 34(4) 495-502, doi:10. 4012/dmj.2014-293.

3. Lauvahutanon S, Takahashi H, Shiozawa M, Iwasaki N, Asakawa Y, Oki M, Finger WJ, \& Arksornnukit M (2014) Mechanical properties of composite resin blocks for CAD/ CAM Dental Materials Journal 33(5) 705-710, doi:10. 4012/dmj.2014-208.

4. Ruse ND, \& Sadoun MJ (2014) Resin-composite blocks for dental CAD/CAM applications Journal of Dental Research 93(12) 1232-1234, doi:10.1177/0022034514553976.

5. Coldea A, Swain MV, \& Thiel N (2013) Mechanical properties of polymer-infiltrated-ceramic-network materials Dental Materials 29(4) 419-426, doi:10.1016/j.dental. 2013.01.002.

6. Nguyen JF, Ruse D, Phan AC, \& Sadoun MJ (2013) Hightemperature-pressure polymerized resin-infiltrated ceramic networks Journal of Dental Research 93(1) 62-67, doi:10.1177/0022034513511972.

7. Leung BTW, Tsoi JKH, Matinlinna JP, \& Pow EHN (2015) Comparison of mechanical properties of three machinable ceramics with an experimental fluorophlogopite glass ceramic Journal of Prosthetic Dentistry 114(3) 440-446, doi:10.1016/j.prosdent.2015.02.024. 
8. Della Bona A, Corazza PH, \& Zhang Y (2014) Characterization of a polymer-infiltrated ceramic-network material Dental Materials 30(5) 564-569, doi:10.1016/j.dental. 2014.02.019.

9. Krämer N, Lohbauer U, \& Frankenberger R (2000) Adhesive luting of indirect restorations American Journal of Dentistry 13(Spec No) 60D-76D.

10. D'Arcangelo C, \& Vanini L (2007) Effect of three surface treatments on the adhesive properties of indirect composite restorations Journal of Adhesive Dentistry 9(3) 319-326.

11. Peumans M, Hikita K, De Munck J, Van Landuyt K, Poitevin A, Lambrechts P, \& Van Meerbeek B (2007) Effects of ceramic surface treatments on the bond strength of an adhesive luting agent to CAD-CAM ceramic Journal of Dentistry 35(4) 282-288, doi:10.1016/ j.jdent.2006.09.006.

12. Krämer N, Reinelt C, Richter G, Petschelt A, \& Frankenberger R (2009) Nanohybrid vs. fine hybrid composite in Class II cavities: Clinical results and margin analysis after four years Dental Materials 25(6) 750-759, doi:10.1016/j.dental.2008.12.003.

13. Spitznagel FA, Horvath SD, Guess PC, \& Blatz MB (2014) Resin bond to indirect composite and new ceramic/ polymer materials: A review of the literature Journal of Esthetic and Restorative Dentistry 26(6) 382-393, doi:10. 1111/jerd.12100.

14. Elsaka SE (2014) Bond strength of novel CAD/CAM restorative materials to self-adhesive resin cement: The effect of surface treatments Journal of Adhesive Dentistry 16(6) 531-540, doi:10.3290/j.jad.a33198.

15. Frankenberger R, Hartmann V, Krech M, Krämer N, Reich S, Braun A, \& Roggendorf M. (2015) Adhesive luting of new CAD/CAM materials International Journal of Computerized Dentistry 18(1) 9-20.

16. Poitevin A, De Munck J, Van Landuyt K, Coutinho E, Peumans M, Lambrechts P, \& Van Meerbeek B (2008) Critical analysis of the influence of different parameters on the microtensile bond strength of adhesives to dentin Journal of Adhesive Dentistry 10(1) 7-16.

17. $\mathrm{R}$ Core Team (2013) $R$ : A Language and Environment for Statistical Computing R Foundation for Statistical Computing, Vienna, Austria.

18. Loomans BA, Cardoso MV, Opdam NJM, Roeters FJM, De Munck J, Huysmans MC, \& Van Meerbeek B (2011) Surface roughness of etched composite resin in light of composite repair Journal of Dentistry 39(7) 499-505, doi: 10.1016/j.jdent.2011.04.007.

19. Loomans BA, Vivan Cardoso M, Roeters FJM, Opdam NJM, De Munck J, Huysmans MC, \& Van Meerbeek B (2011) Is there one optimal repair technique for all composites? Dental Materials 27(7) 701-709, doi:10. 1016/j.dental.2011.03.013.

20. Brentel AS, Özcan M, Valandro LF, Alarça LG, Amaral R, \& Bottino MA (2007) Microtensile bond strength of a resin cement to feldpathic ceramic after different etching and silanization regimens in dry and aged conditions Dental Materials 23(11) 1323-1331, doi:10.1016/j.dental.2006.11. 011.
21. Özcan M, Valandro LF, Amaral R, Leite F, \& Bottino MA (2009) Bond strength durability of a resin composite on a reinforced ceramic using various repair systems Dental Materials 25(12) 1477-1483, doi:10.1016/j.dental.2009.06. 020.

22. Ellakwa A, Shortall A, Burke F, \& Marquis P (2003) Effects of grit blasting and silanization on bond strengths of a resin luting cement to Belleglass HP indirect composite American Journal of Dentistry 16(1) 53-57.

23. Yoshida K, Kamada K, \& Atsuta M (2001) Effects of two silane coupling agents, a bonding agent, and thermal cycling on the bond strength of a CAD/CAM composite material cemented with two resin luting agents Journal of Prosthetic Dentistry 85(2) 184-189, doi:10.1067/mpr. 2001.113628.

24. Lung CYK, \& Matinlinna JP (2012) Aspects of silane coupling agents and surface conditioning in dentistry: An overview Dental Materials 28(5) 467-477, doi:10.1016/j. dental.2012.02.009.

25. El Zohairy AA, De Gee AJ, Hassan FM, \& Feilzer AJ (2004) The effect of adhesives with various degrees of hydrophilicity on resin ceramic bond durability Dental Materials 20(8) 778-787, doi:10.1016/j.dental.2003.05. 010.

26. Kirmali O, Barutcugil C, Harorli O, Kapdan A, \& Er K (2015) Resin cement to indirect composite resin bonding: Effect of various surface treatments Scanning $\mathbf{3 7 ( 2 )}$ 89-94, doi:10.1002/sca.21183.

27. Campos F, Almeida CS, Rippe MP, de Melo RM, Valandro LF, \& Bottino MA (2015) Resin bonding to a hybrid ceramic: Effects of surface treatments and aging Operative Dentistry 41(2) 171-178, doi:10.2341/15-057-L.

28. Özcan M, Allahbeickaraghi A, \& Dündar M (2012) Possible hazardous effects of hydrofluoric acid and recommendations for treatment approach: A review Clinical Oral Investigations 16(1) 15-23, doi:10.1007/ s00784-011-0636-6.

29. Scherrer SS, Cesar PF, \& Swain MV (2010) Direct comparison of the bond strength results of the different test methods: A critical literature review Dental Materials 26(2) e78-e93, doi:10.1016/j.dental.2009.12.002.

30. Lise DP, Perdigão J, Van Ende A, Zidan O, \& Lopes G (2015) Microshear bond strength of resin cements to lithium disilicate substrates as a function of surface preparation Operative Dentistry 40(5) 524-532, doi:10. 2341/14-240-L.

31. Nagai T, Kawamoto Y, Kakehashi Y, \& Matsumura H (2005) Adhesive bonding of a lithium disilicate ceramic material with resin-based luting agents Journal of Oral Rehabilitation 32(8) 598-605, doi:10.1111/j.1365-2842. 2005.01464.x.

32. Spohr A, Sobrinho L, Consani S, Sinhoreti M, \& Knowles $J$ (2003) Influence of surface conditions and silane agent on the bond of resin to IPS Empress 2 ceramic International Journal of Prosthodontics 16(3) 277-282.

33. Kalavacharla V, Lawson N, Ramp L, \& Burgess J (2014) Influence of etching protocol and silane treatment with a universal adhesive on lithium disilicate bond strength Operative Dentistry 40(4) 372-378, doi:10.2341/14-116-L. 